\title{
Bioorthogonal Chemical Handle for Tracking Multifunctional Nanoparticles
}

\author{
Sascha N. Goonewardena, ${ }^{*[a, b]}$ Hong Zong, ${ }^{[b]}$ Pascale R. Leroueil, ${ }^{[b]}$ and James R. Baker, Jr. ${ }^{[b]}$
}

\begin{abstract}
Nanoparticle technologies have tremendous potential in clinical medicine. To fully realize this potential, one must further understand how nanoparticles interact with biological systems. Typically, reporters that are conjugated to nanoparticles during synthesis are used to monitor the nanoparticles in biological systems. Unfortunately, conjugating reporters to nanoparticles complicates the synthesis and the reporter itself may alter the nanoparticle properties. To address these challenges, a coppercatalyzed azide-alkyne cycloaddition strategy has been developed to functionalize nanoparticles with fluorescent reporters
\end{abstract}

after they have been delivered to biological systems. Using polyamidoamine dendrimers as model nanoparticles, the utility of this strategy is shown in several biological systems including a cancer cell model, primary immune cells, and a murine model of inflammation. This reporter strategy simplifies the synthesis without sacrificing the ability to monitor the nanoparticle conjugates. It is expected that this bioorthogonal reporter strategy can be used to understand nanoparticle interactions in biological systems, which will facilitate the translation of these technologies to the clinics.

\section{Introduction}

Owing to their small size, nanoparticles have unique properties compared with the bulk form of the same material. Despite the speed of development of nanoparticles, relatively little is known about their interactions with living systems. To further understand the nature of nanoparticle interactions with biological systems, nanoparticles must be engineered with reporters that can be used to comprehend these interactions. Unfortunately, engineering nanoparticles with reporters can be challenging.

The most common strategy used to monitor nanoparticles is to conjugate reporters to the nanoparticle scaffold during synthesis. The reporter can then be used to monitor the nanoparticle in biological systems. Although this strategy is useful, it has drawbacks that limit its utility. First, conjugating reporters to nanoparticles complicates the synthesis because of the additional steps and purifications. Second, many reporters themselves have distinct chemical characteristics that, if conjugated to the nanoparticle, can alter the properties of the conjugate. ${ }^{[1]}$ Clearly, improved nanoparticle monitoring strategies are needed to better understand how nanoparticles interact with biological systems. In particular, the development of strategies

\section{[a] Dr. S. N. Goonewardena}

Internal Medicine, Division of Cardiovascular Medicine

University of Michigan, CVC Room 2547, 1500 E. Medical Center Drive SPC 5853, Ann Arbor, MI 48109-5853 (USA)

E-mail:sngoonew@med.umich.edu

[b] Dr. S. N. Goonewardena, ${ }^{+}$Dr. H. Zong, ${ }^{+}$Dr. P. R. Leroueil, Prof. J. R. Baker, Jr. Michigan Nanotechnology Institute for Medicine and Biological Sciences University of Michigan, Room 9220C MSRBIII, 1150 W. Medical Center Drive Ann Arbor, MI 48109 (USA)

$\left.{ }^{[}\right]$These authors contributed equally to this work.

Supporting information for this article is available on the WWW under http://dx.doi.org/10.1002/cplu.201300007. that are simple, rapid, and sensitive and do not require complicated syntheses or alter the properties of the nanoparticle remains highly desirable.

Recently, bioorthogonal reactions have emerged as promising tools for chemistry and biological applications. The [3+2] cycloaddition between azides and terminal alkynes is one of the first bioorthogonal reactions and was described by Huisgen more than four decades ago. ${ }^{[2]}$ Although this reaction is thermodynamically favorable, it requires harsh reaction conditions that limit its use. To overcome some of these obstacles, Sharpless and colleagues used a copper catalyst to accelerate the reaction. This modification greatly expanded the utility of this reaction and has led to more extensive applications in organic and polymer chemistry. ${ }^{[3]}$ Additionally, because of the efficiency, selectivity, and mild reaction conditions, this class of reactions is well suited for profiling endogenous macromolecules in biological systems. For example, the copper-catalyzed azide-alkyne cycloaddition (CUAAC) has been used for in situ conjugation of chemical reporters to monitor DNA synthesis, metabolite flux, and protein trafficking. ${ }^{[4]}$

These studies demonstrated that the CuAAC reaction can be used to monitor biomolecules and overcomes many of the limitations of traditional reporter strategies. Synthetic nanoparticles are similar in many respects to biomolecules, so we hypothesized that the CUAAC reaction could be used to conjugate reporters to nanoparticles after their delivery to biological systems. To explore this possibility, we synthesized dendrimer scaffolds with alkyne chemical handles that could be used for downstream CUAAC functionalization. This approach not only allowed us to engineer multifunctional dendrimer conjugates, but also provided a chemical handle that could be reacted with azide-modified fluorescent reporters in biological systems. We evaluated this reporter strategy in several biological sys- 


\section{CHEMPLUSCHEM}

tems, including cancer and immune cell models and an animal model of inflammation. We anticipate that this approach will simplify the design of multifunctional nanoparticles and can be used to further understand the biological behavior of nanoparticles, which will speed their translation to the clinics.

\section{Results and Discussion}

\section{Comparison of dendrimer CuAAC reaction efficiencies}

Figure 1 summarizes the strategy to functionalize dendrimers with chemical reporters in biological systems. To evaluate this strategy, we first synthesized G5-Alkyne 2 with 12.3 alkyne handles per dendrimer (Figure S1 in the Supporting Information). After synthesizing G5Alkyne 2, the other dendrimer conjugates were functionalized

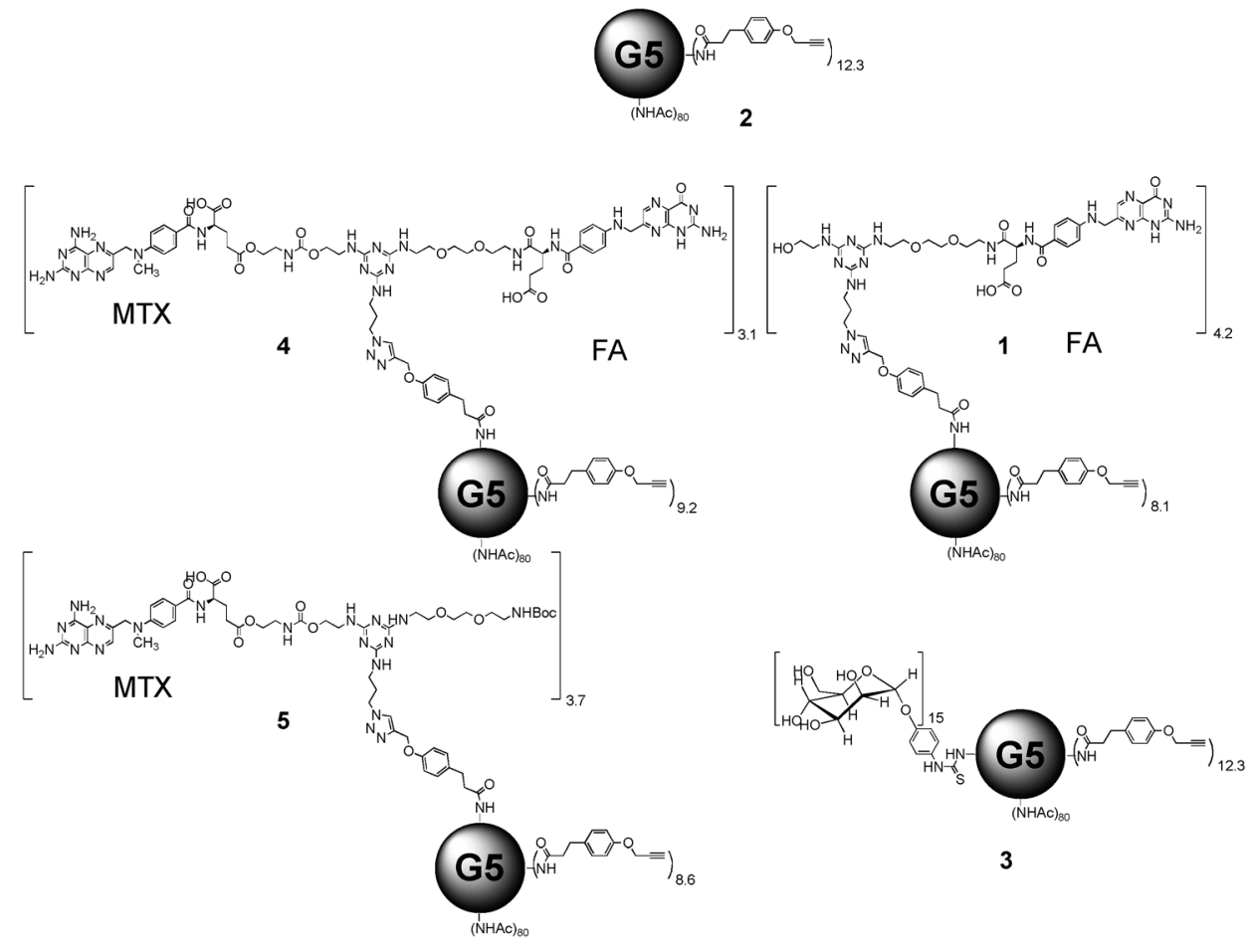

Scheme 1. G5-FA-Alkyne 1, G5-Alkyne 2, G5-Mannose-Alkyne 3, G5-FA-MTX-Alkyne 4, and G5-MTX-Alkyne 5.

$\mathrm{G} 5=$ generation 5 polyamidoamine $(\mathrm{PAMAM})$ dendrimer, $\mathrm{FA}=$ folic acid, MTX $=$ methotrexate, Boc $=$ tert-butoxycarbonyl. with azide-derivatized triazine rings by using the CuAAC reaction (Scheme 1). For the reporter strategy to be useful, it is important that the CuAAC reaction efficiencies of the different dendrimer conjugates are similar so

that the conjugated reporter reflects the amount of dendrimer present rather than differences in reaction efficiencies. To confirm that the reaction efficiencies were similar between dendri-

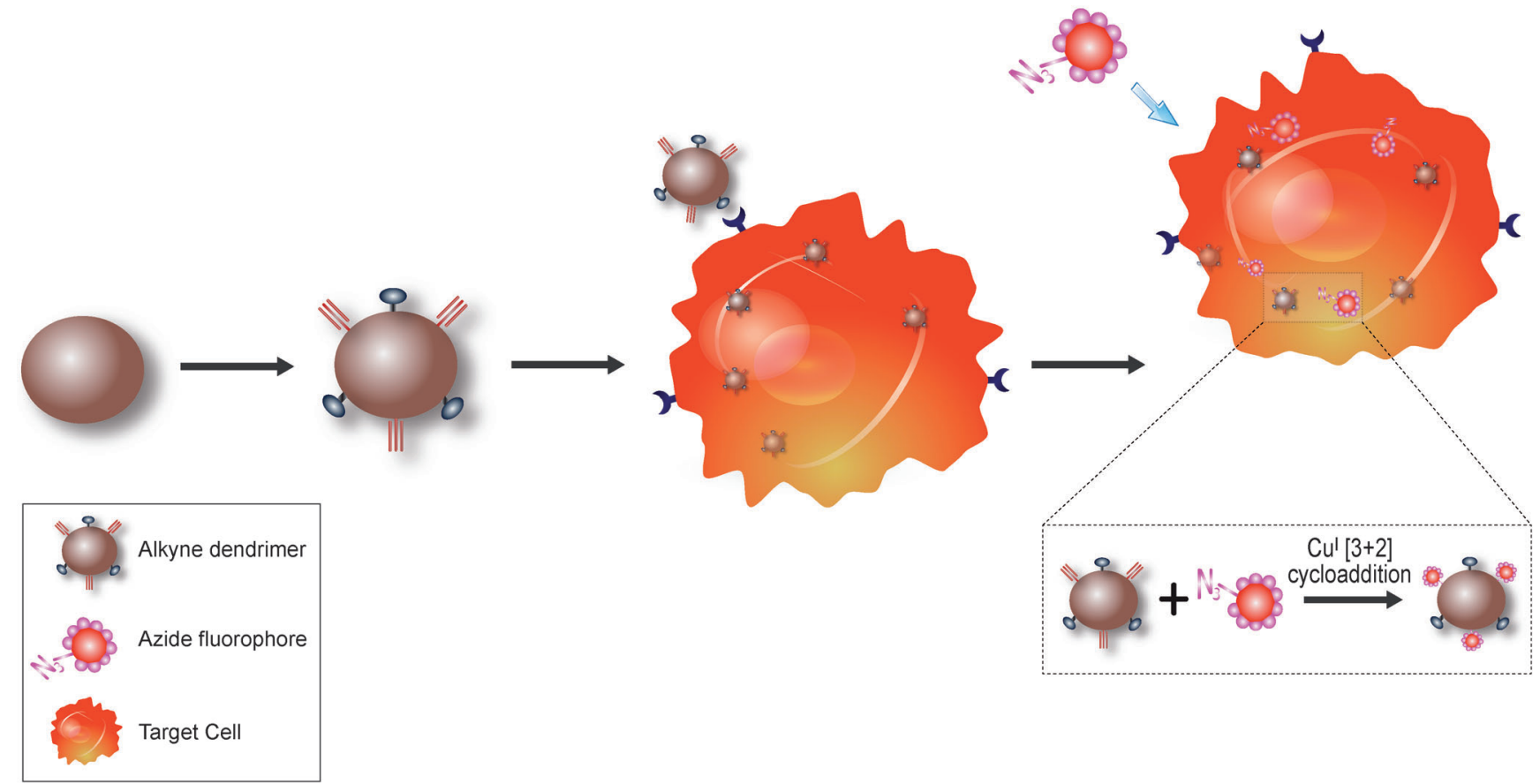

Figure 1. Schematic of the bioorthogonal reporter strategy for monitoring dendrimer conjugates. Dendrimers are engineered with surface alkyne handles that can be further functionalized. The dendrimer conjugates are then delivered to biological targets. The dendrimer alkyne handles are reacted in situ with azido-fluorescent reporters through the CuAAC reaction. 
mer conjugates, we used a "click" fluorescence assay that consists of the ligation of a quenched 3-azido-7-hydroxycoumarin reporter to alkynefunctionalized dendrimer conjugates to form a triazole-linked adduct. After the ligation, there is an increase in fluorescence intensity that reflects the CuAAC reaction efficiency. ${ }^{[5]}$ To evaluate the CuAAC reaction efficiencies, we reacted equal amounts of the 3-azido-7-hydroxycoumarin reporter with the different alkyne-functionalized dendrimer conjugates and assessed the resulting fluorescence signal. After 1 hour, no significant differences in fluorescence intensities between the different dendrimer conjugates were found (Table S1). These results confirm that the CuAAC reaction efficiencies are similar for the different dendrimer conjugates.

\section{In vitro evaluation of CUAAC reporter strategy}

We then evaluated the CuAAC reporter strategy in a cancer cell model. We and others have shown that FA-functionalized dendrimers internalize through the folate receptor (FR) in the $\mathrm{KB}$ cell line. ${ }^{[6]}$ To evaluate the reporter strategy, we incubated G5-FA-Alkyne 1 and the nontargeted control G5-Alkyne 2 with KB cells for 1 hour at $37^{\circ} \mathrm{C}$. After incubation, the cells were processed and AF647 dye (see the Experimental Section) was conjugated to the internalized dendrimer conjugates by using the CuAAC reaction. Similar to traditional, fluorophore-labeled FA-dendrimer conjugates, the G5-FA-Alkyne 1 demonstrated dose- and time-dependent uptake into KB cells (Figure 2 and Figure S3). The uptake of G5FA-Alkyne 1 could be blocked by treatment with FA $(100 \mu \mathrm{M})$, thus confirming the FR specificity of the FA-targeted dendrimer conjugates (Figure 2). The cells treated with the nontargeted G5-Alkyne 2 showed no evidence of uptake, with fluorescence signals similar to those of the untreated cells (Figure 2). Importantly, in the absence of the copper catalyst, there was no evidence of increased fluorescence signal relative to controls, which suggested strongly that the fluorescence signal is from ligation of the fluorescent reporters with the internalized dendrimer conjugates (Figure S4).
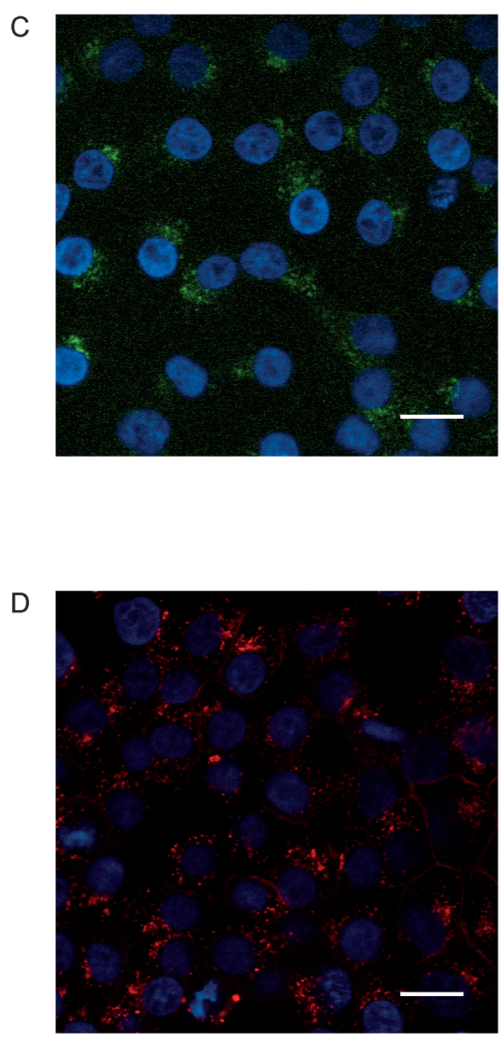

Figure 2. In vitro demonstration of bioorthogonal reporter in a cancer cell model. A) KB cells were incubated with with AF647 by using the CuAAC reaction and analyzed by flow cytometry. B) G5-FA-Alkyne 1 uptake was inhibited analyzed by flow cytometry. C) KB cells were incubated with $30 \mathrm{nM}$ G5-FA-Alkyne 1 and stained with $4^{\prime}, 6$-diamidino-2-phenylindole (DAPI; blue), and internalized dendrimer conjugates were reacted with AF488 (green).

D) KB cells were incubated with the same G5-FA-Alkyne 1 conjugate as in (C). The cells were then stained with DAPI (blue) and reacted with AF555 (red). Scale bars: $20 \mu \mathrm{m}$. Results are representative of three independent experiments. $\mathrm{MFI}=$ median fluorescence intensity. No $\mathrm{Tx}=$ no treatment.

\section{Evaluation of CuAAC reporter strategy with microscopy}

After showing the utility of the reporter strategy with flow cytometry, we explored its utility in microscopy applications. Although flow cytometry provides valuable information, it does not capture spatial information and relationships. Unfortunately, the use of fluorescent reporters can be more challenging in microscopy applications because of the complex processing protocols and the incompatibility of some fluorescent reporters. ${ }^{[7]}$ Using a protocol that preserves cell monolayers, we evaluated the ability of the CuAAC reporter strategy to functionalize cell-internalized dendrimer conjugates that could be analyzed by confocal microscopy. KB cells were incubated with either G5-FA-Alkyne 1 or the nontargeted control G5-Alkyne 2 for 1 hour at $37^{\circ} \mathrm{C}$. After the treatment, the cells were processed and the internalized dendrimer conjugates were reacted with either AF488 or AF555 reporters by using the CUAAC reaction. In agreement with the flow cytometry results, the internalized G5-FA-Alkyne 1 conjugates could be reacted with azide-modified fluorescent reporters through the CuAAC reac- 
tion and visualized by confocal microscopy (Figure 2). Additionally, we demonstrate the flexibility of this strategy by using different fluorescent reporters without having to synthesize new dendrimer conjugates.

\section{Validation of CuAAC reporter strategy in a primary cell model}

To evaluate the generalizability of the CuAAC reporter strategy, we engineered $\alpha$-D-mannose dendrimer conjugates (G5-Mannose-Alkyne 3) that target tumor-associated macrophages (TAMs) through the macrophage mannose receptor (MMR). $\alpha$ D-Mannose is a high-affinity ligand for the MMR, a type 1 membrane receptor that is present on TAMs and is being investigated for immune therapeutics. ${ }^{[8]}$ The MMR is induced in cytokine-polarized bone-marrow-derived macrophages (BMDMs), thereby providing another receptor-ligand system to test the in situ CuAAC reporter strategy..$^{[9]}$ The TAMs were incubated with G5-Mannose-Alkyne 3 and the nontargeted G5Alkyne 2 for $6 \mathrm{~h}$ at $37^{\circ} \mathrm{C}$. The TAMs were then processed and the internalized dendrimer conjugates were reacted with AF647 by using the CuAAC reaction and then analyzed by flow cytometry. The TAMs treated with G5-Mannose-Alkyne 3 showed an increase in fluorescence signal compared with TAMs treated with G5-Alkyne 2 and nonpolarized BMDMs, thus confirming the receptor-specific internalization of G5-MannoseAlkyne 3 (Figure 3). The cell-specific uptake and detection of mannose-functionalized alkyne dendrimers by using the
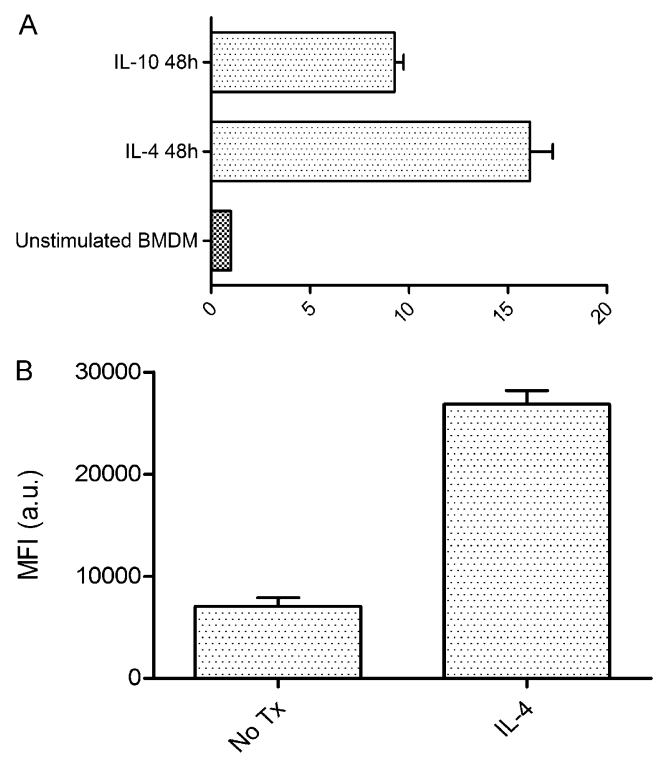

Figure 3. In vitro evaluation of bioorthogonal reporter in primary immune cells. A) MMR was induced in BMDMs stimulated with interleukin 4 (IL-4) and IL-10 as assessed by real-time quantitative reverse-transcription polymerase chain reaction (qRT-PCR). B) IL-4- and IL-10-stimulated BMDMs were incubated with $30 \mathrm{~nm}$ G5-Mannose-Alkyne 3 and G5-Alkyne 2 for $6 \mathrm{~h}$. BMDMs were harvested and internalized dendrimer conjugates were reacted with AF647 and analyzed by flow cytometry. G5-Mannose-Alkyne 3 internalizes into BMDMs stimulated with IL-4 and IL-10 but not into unstimulated BMDMs. Results are representative of two independent experiments. No $T x=$ no treatment.
CuAAC reporter strategy confirms the efficacy of this approach for a broad spectrum of receptor-ligand systems, nanoparticles, and cell types.

\section{Evaluation of CuAAC reporter strategy to monitor multifunc- tional dendrimer therapeutics}

One important application for the in situ CuAAC reporter strategy is to monitor multifunctional nanoparticles without having to synthesize distinct, reporter-labeled conjugates. Typically, nanoparticle therapeutics must be engineered with reporters during their fabrication to allow monitoring in biological systems. Engineering of nanoparticles with these reporters complicates their synthesis and the conjugated reporter itself may alter the properties of the nanoparticle. To evaluate the utility of the CUAAC reporter strategy to monitor nanoparticle therapeutics, we compared the binding properties of two closely related dendrimer therapeutics (G5-FA-MTX-Alkyne 4 and G5MTX-Alkyne 5) in a cancer cell model. We and others have shown that decorating the surface of nanoparticles with targeting ligands enhances the binding avidity compared with the monovalent targeting ligand. ${ }^{[10]}$ Although both FA and MTX bind the FR, MTX has a 100-fold lower affinity than FA. ${ }^{[1]}$ Several studies using analytical techniques have demonstrated that conjugating multiple MTX molecules to the dendrimer surface enhances the binding affinity through multivalent receptor interactions. ${ }^{[12]}$ However, these findings have not been evaluated systematically in biological systems because of the difficulties in engineering reporter-labeled nanoparticle therapeutics. Using the CuAAC reporter strategy to monitor the dendrimer conjugates, we explored the binding properties of dendrimer therapeutics in a culture model. We used the alkyne handles to further functionalize the dendrimer conjugates during the synthesis and also to attach the fluorescent reporters in biological systems, so the syntheses were greatly simplified without losing the ability to monitor the dendrimer conjugates. KB cells were treated with G5-FA-MTX-Alkyne 4 and G5MTX-Alkyne 5 for 1, 4, and $24 \mathrm{~h}$. After the incubation, the cells were processed and the internalized dendrimer conjugates were reacted with azide reporters and analyzed by flow cytometry as described above. The G5-FA-MTX-Alkyne 4 and G5MTX-Alkyne 5 both showed FR-specific uptake in the KB cells. Interestingly, $\mathbf{4}$ was internalized more efficiently than $\mathbf{5}$ at all of the time points examined (Figure 4). These findings build upon our prior studies and suggest that the rate and total amount of the G5-MTX-Alkyne 5 internalized are much less than G5-FAMTX-Alkyne 4. These types of biological studies on nanoparticle properties are difficult to perform because of the complicated synthetic schemes needed to engineer comparable reporter-labeled conjugates and the necessary controls. The modularity of our synthetic approach and the strengths of the CuAAC reporter strategy address some of these difficulties and enable more extensive evaluations of the interactions between nanoparticles and biological systems. 
A

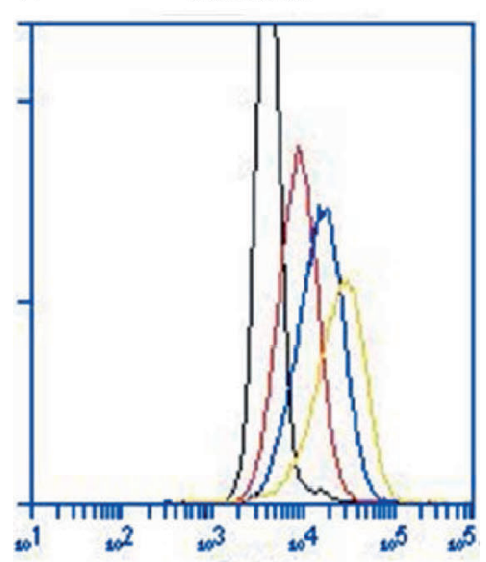

G5MTX

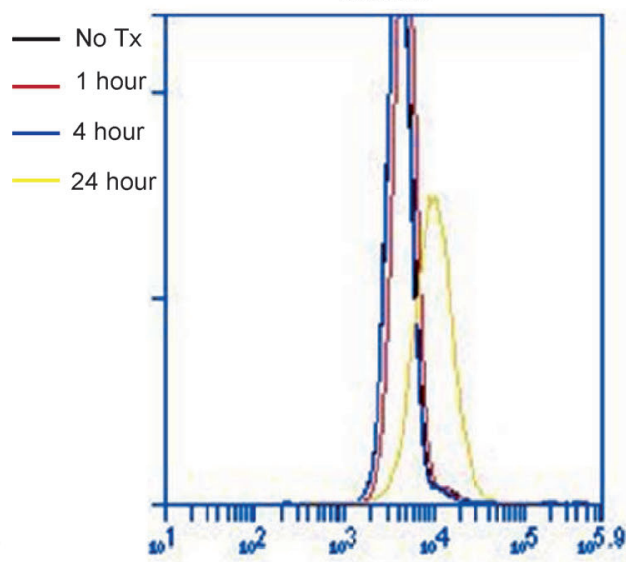

B

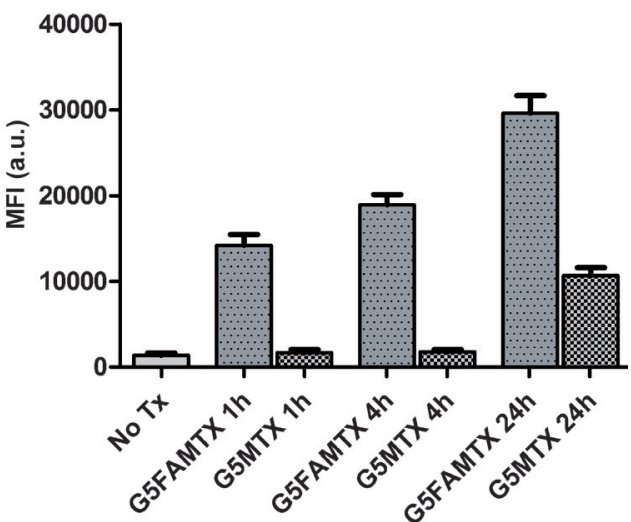

Figure 4. Monitoring dendrimer therapeutics by using the bioorthogonal reporter strategy. A) KB cells were incubated with 30 nM G5-FA-MTX-Alkyne 4 and G5-MTX-Alkyne 5 for the indicated times. Cells were harvested at various time points and the internalized dendrimer therapeutics were reacted with AF647 by using the CUAAC. B) G5-FA-MTX-Alkyne 4 internalizes more rapidly and in a greater amount than G5-MTX-Alkyne 5. Results are representative of three independent experiments. No $T x=$ no treatment.

\section{Validation of CuAAC reporter strategy in an animal model of inflammation}

After confirming the efficacy of our CuAAC reporter strategy in vitro, we evaluated its utility to monitor nanoparticles in vivo. One of the major obstacles slowing the translation of nanomaterials to the clinics is a limited understanding of their biological properties in vivo. ${ }^{[13]}$ To evaluate the CuAAC reporter strategy in vivo, we used a murine model of inflammation and examined the uptake and trafficking of G5-FA-Alkyne 1. We and others have shown that thioglycollate administered intraperitoneally (IP) induces recruitment of folate receptor beta $\left(\mathrm{FR} \beta^{+}\right)$ inflammatory macrophages that can bind and internalize FA-targeted dendrimers. ${ }^{[14]}$ On day 1 , mice $(n=2)$ were treated with thioglycollate to induce peritoneal inflammation and recruitment of $\mathrm{FR} \beta^{+}$macrophages. On day 3, mice were administered $35 \mathrm{mg} \mathrm{kg}^{-1}$ G5-FA-Alkyne 1, G5Alkyne 2, or a vehicle control IP. On day 4, peritoneal macrophages, submandibular lymph nodes, and mesenteric lymph nodes were isolated and processed for single-cell suspensions. The cell suspensions and the internalized dendrimers were reacted with azide-modified fluorescent reporters by using the CuAAC reaction and analyzed by flow cytometry as described above. As expected, the G5-FA-Alkyne 1 conjugates were found in the $\mathrm{FR} \beta^{+}$peritoneal macrophages and the $\mathrm{FR} \beta^{+}$ macrophages in the secondary lymph organs draining the peritoneal cavity (mesenteric lymph nodes; Figure 5). In contrast, secondary lymph organs that do not drain the peritoneal
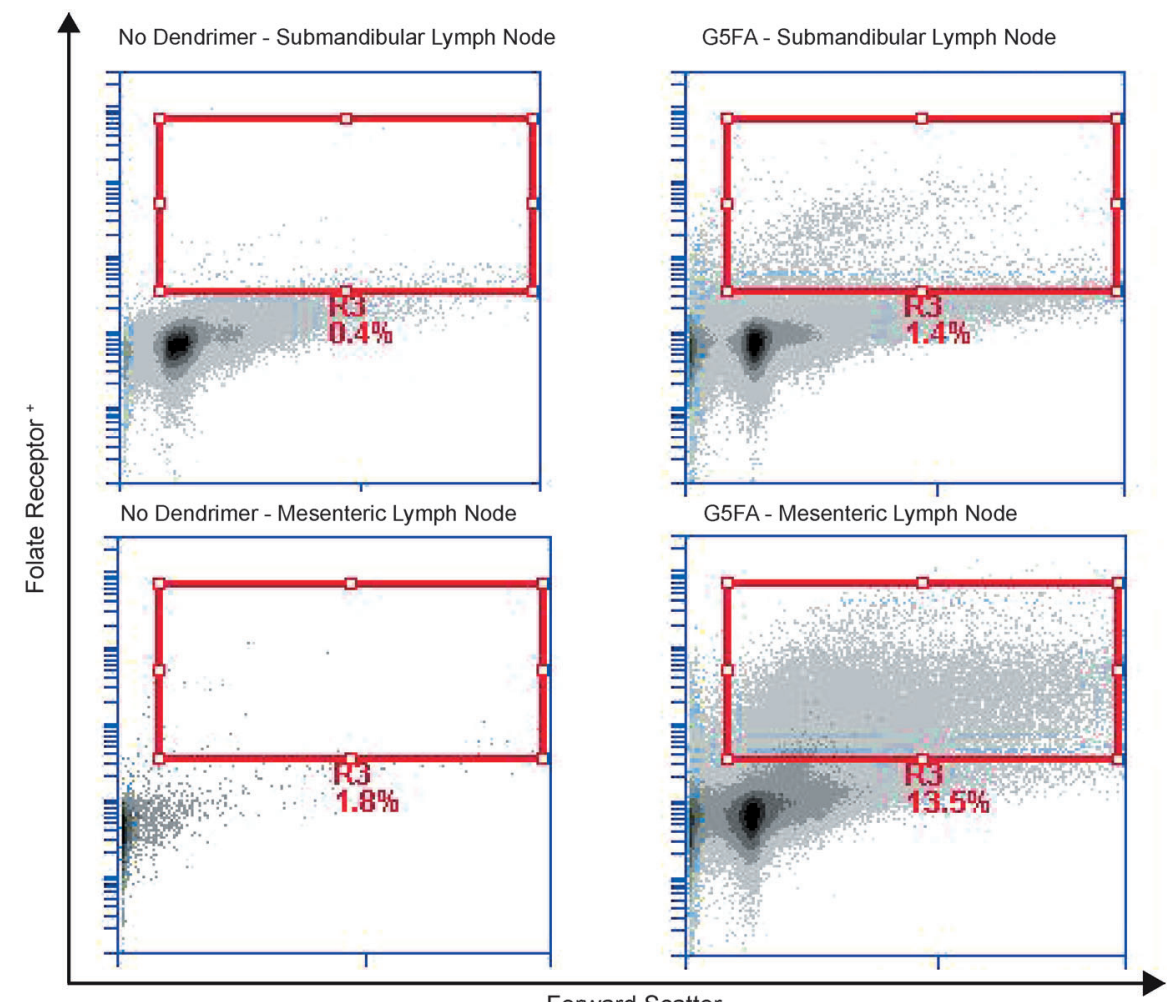

Forward Scatter

Figure 5. In vivo monitoring of dendrimer conjugates with the bioorthogonal reporter strategy. Mice were stimulated with thioglycollate IP. Three days after stimulation, mice were treated IP with G5-FA-Alkyne 1 (200 $\mu$ L, $35 \mathrm{mg} \mathrm{kg}^{-1}$ ). On day 4, peritoneal macrophages, spleen, cervical lymph nodes, and mesenteric lymph nodes were isolated and internalized dendrimers were reacted with AF647 and analyzed by flow cytometry. Results show more G5-FA-Alkyne 1 uptake into macrophages from the mesenteric lymph node than the submandibular lymph node, consistent with the immune trafficking of $\mathrm{FR} \beta^{+}$peritoneal macrophages. 
cavity (submandibular lymph nodes) showed no evidence of macrophages that had internalized the G5-FA-Alkyne 1, consistent with the known trafficking patterns of the peritoneal macrophages. Additionally, the peritoneal macrophages from stimulated mice had a much higher uptake of G5-FA-Alkyne 1 than the control mice, consistent with the high prevalence of $\mathrm{FR} \beta^{+}$macrophages following activation with thioglycollate (data not shown). These results show that the CuAAC reporter strategy can be used to monitor nanoparticle trafficking in animal models.

\section{Conclusion}

In summary, we have developed a bioorthogonal, copper-catalyzed azide-alkyne cycloaddition reporter strategy to conjugate fluorescent reporters to nanoparticles in biological systems. To our knowledge, this is the first description of in situ conjugation of reporters to synthetic nanoparticles to track nanoparticles in vitro and in vivo. The nanoparticle alkyne handles can be further functionalized after delivery to biological systems, so there is no need to synthesize distinct reporter-labeled conjugates to monitor their trafficking. We believe that this strategy provides an alternative to traditional labeling approaches while providing the flexibility for multiple reporters as well as more detailed biological evaluations of multifunctional nanoparticles. We envision that this bioorthogonal reporter strategy will be applicable to a broad spectrum of nanomaterials and opens the door for mechanistic studies on nanoparticle-biology interactions that will facilitate their translation to the clinics.

\section{Experimental Section}

\section{Materials}

All solvents and chemicals were reagent-grade quality and were purchased from Sigma-Aldrich. Generation 5 (G5) polyamidoamine (PAMAM) dendrimer was purchased from Dendritech, Inc., Midland, Michigan. Alexa Fluor 488 azide (AF488), Alexa Fluor 555 azide (AF555), Alexa Fluor 647 azide (AF647), trypsin-EDTA, and phosphate-buffered saline (PBS) were purchased from Life Technologies (Carlsbad, CA, USA). All materials were used without further purification unless otherwise noted.

\section{General methods}

All ${ }^{1} \mathrm{H}$ NMR spectra were measured on a $500 \mathrm{MHz}$ Varian NMR system equipped with a multinuclear $5 \mathrm{~mm}$ probe. Mass spectra were obtained on a Waters 1525 mass spectrometer. Silica gel 60 had particle size $0.040-0.063 \mathrm{~mm}$ and $230-400 \mathrm{mesh}$; thin-layer chromatography was performed using Whatman adsorption plates, $60 \AA$ silica gel, $250 \mu \mathrm{m}$ layer thickness. Deionized water (18.2 M $\Omega$ ) was made with a Nanopure Infinity DI water system.

\section{Characterization of dendrimer conjugates}

The dendrimer conjugates and their structures are shown in Scheme 1. The number of ligands attached to the dendrimer was derived from the integration of the methyl protons of the terminal acetyl groups to the aromatic protons from the conjugated ligands (e.g., FA and MTX) as described previously. ${ }^{[15]}$ The number of acetyl groups per dendrimer was determined by computing the total number of end groups from the gel permeation chromatography average molecular weight and potentiometric titration data for G5$\mathrm{NH}_{2}(100 \%)$ as described previously. ${ }^{[15,16]}$ The total number of end groups was applied to the ratio of primary amines to acetyl groups, obtained from the ${ }^{1} \mathrm{H}$ NMR data of the partially acetylated dendrimer, to compute the average number of acetyl groups per dendrimer.

Ultraperformance liquid chromatography (UPLC) analysis was performed on a Waters Acquity Peptide Mapping System equipped with a Waters photodiode array detector, a column manager, and a sample manager. The instrument was controlled by Empower 3 software. For characterization, calibration, and quantitation studies, dendrimers were separated on an Acquity BEH C18 column (100x $2.1 \mathrm{~mm}, 1.7 \mu \mathrm{m})$. The analysis was performed by using gradient elution beginning with 99:1 (v/v) water/acetonitrile (MeCN) and reaching 20:80 water/MeCN in $13.4 \mathrm{~min}$. Trifluoroacetic acid ( 0.14 wt \% concentration) was added to the water as well as MeCN as a counter ion. The gradient was then equilibrated back to the starting conditions in the next $1.0 \mathrm{~min}$. The flow rate was maintained at $0.208 \mathrm{~mL} \mathrm{~min}^{-1}$, the column temperature at $35^{\circ} \mathrm{C}$, and the concentration of dendrimer at $1.0 \mathrm{mg} \mathrm{mL}^{-1}$.

\section{Synthesis of dendrimer conjugates}

All conjugates and intermediates were purified by ultrafiltration using 10000 molecular weight cutoff centrifugal filtration devices (Millipore). Purification consisted of ten cycles (20 min at $4800 \mathrm{rpm}$ ) using PBS (five cycles) and deionized water (five cycles). All conjugates and intermediates were characterized by MALDI-TOF, UPLC, and ${ }^{1} \mathrm{H}$ NMR spectroscopy.

Synthesis of FA and MTX dendrimer conjugates: G5-FA-Alkyne 1, G5-Alkyne 2, G5-FA-MTX-Alkyne 4, and G5-MTX-Alkyne 5 were synthesized as described previously. ${ }^{[17,18]}$ G5-FA-Alkyne 1 (yellow solid): ${ }^{1} \mathrm{H}$ NMR integration determined the mean number of FA units attached to be 4.2; MALDI-TOF mass 33059. G5-Alkyne 2 (white solid): By ${ }^{1} \mathrm{H}$ NMR integration, the mean number of acetyl groups per dendrimer was 80.1 ; the mean number of alkyne ligands per dendrimer was 12.3; MALDI-TOF mass 32774. G5-FA-MTX-Alkyne 4 (yellow solid): ${ }^{1} \mathrm{H}$ NMR integration determined the mean number of triazine-MTX-FA units attached to be 3.1; MALDI-TOF mass 35094. G5-MTX-Alkyne 5 (yellow solid): 'H NMR integration determined the mean number of MTX units attached to be 3.7; MALDI-TOF mass 34266 .

Synthesis of G5-Mannose-Alkyne 3: $\alpha$-D-Mannopyranosylphenyl isothiocyanate $(1.5 \mathrm{mg}, 4.8 \mu \mathrm{mol})$ in dimethyl sulfoxide (DMSO; $100 \mu \mathrm{L}$ ) was added to a solution of G5-Alkyne 2 (10.5 mg, $0.32 \mu \mathrm{mol})$ and $\mathrm{N}, \mathrm{N}$-diisopropylethylamine $(1.2 \mathrm{mg}, 9.3 \mu \mathrm{mol})$ in DMSO $(300 \mu \mathrm{L})$, and the reaction mixture was stirred at room temperature for $24 \mathrm{~h}$ (see the Supporting Information, Scheme S1). The purified product was lyophilized to yield 3 as a white solid (11.4 mg, 95\%). ${ }^{1} \mathrm{H}$ NMR integration determined the mean number of $\alpha$-D-mannose moieties attached to be 11.5; MALDI-TOF mass 36438 .

\section{Cell culture, treatments, and procedures}

The KB cell line was purchased from the American Type Tissue Collection (ATCC, Manassas, VA, USA) and cultured at $37^{\circ} \mathrm{C}$ and $5 \%$ 
$\mathrm{CO}_{2}$ in RPMI 1640 medium (Mediatech, Herndon, VA, USA). The RPMI 1640 medium was supplemented with penicillin $\left(100\right.$ units $\left.\mathrm{mL}^{-1}\right)$, streptomycin $\left(100 \mathrm{mg} \mathrm{mL}^{-1}\right)$, and $10 \%$ heat-inactivated, low-endotoxin fetal bovine serum (FBS; Mediatech, Herndon, VA, USA). To induce high folate receptor $\alpha$ (FR) expression, the KB cells were cultured in FA-depleted RPMI 1640 media for at least 4 days. Animals were handled in accordance with Animal Use Protocol 10260-1. Bone-marrow-derived macrophages (BMDMs) were obtained as described previously. ${ }^{[19]}$ Briefly, bone marrow precursors from female C57BL/6 mice were isolated and matured for 7 days in RPMI 1640 media supplemented with penicillin $\left(100\right.$ units $\left.\mathrm{mL}^{-1}\right)$, streptomycin $\left(100 \mathrm{mg} \mathrm{mL}^{-1}\right)$, and $10 \%$ heat-inactivated low-endotoxin FBS with L-cell conditioned media as a source of macrophage colony-stimulating factor (M-CSF). Following their maturation, BMDMs were polarized with murine recombinant $\mathrm{M}$ CSF $\left(10 \mathrm{ng} \mathrm{mL}^{-1}\right), \mathrm{IL}-4\left(50 \mathrm{ng} \mathrm{mL}^{-1}\right)$, and IL-10 $\left(10 \mathrm{ng} \mathrm{mL}^{-1}\right)$ for $72 \mathrm{~h}$ (Peprotech, Rocky Hill, NJ, USA) to induce the macrophage mannose receptor (MMR). For flow cytometry assessments, cells were seeded in 12-well dishes at a density of 100000 cells per well and cultured for another $24 \mathrm{~h}$ prior to dendrimer treatments. The cells were then incubated with the dendrimer conjugates at $37^{\circ} \mathrm{C}$ for the indicated times. The cells were washed and trypsinized or scraped followed by centrifugation at $1500 \mathrm{rpm}$ for $5 \mathrm{~min}$ to obtain cell pellets. The pellets were washed twice with PBS and then resuspended in PBS with $0.1 \%$ bovine serum albumin (BSA) prior to flow cytometry assessment with an Accuri C6 flow cytometer (Ann Arbor, MI, USA). Flow cytometry data were analyzed by using FlowJo 8.2 software (TreeStar, New Brunswick, NJ, USA).

\section{In situ CuAAC reaction with dendrimer conjugates}

Cells were washed and pelleted as described above. The cell pellets were then fixed in $4 \%$ paraformaldehyde and permeabilized with $10 \%$ saponin in PBS with $0.1 \%$ BSA prior to in situ CuAAC reaction. Fluorescent azide reporters were reacted with dendrimer conjugates for $30 \mathrm{~min}$ with Tris $(100 \mathrm{~mm}, \mathrm{pH} 8.5), \mathrm{CuSO}_{4}(1 \mathrm{~mm})$, fluorescent azide reporters (1-100 $\mu \mathrm{M})$, and ascorbic acid (100 mM). The staining solution was prepared fresh for each experiment. After staining, the cells were washed twice with $10 \%$ saponin/BSA solution and resuspended in PBS/BSA prior to flow cytometry analysis.

\section{Confocal laser scanning microscopy}

KB cells were seeded as described above on glass-bottomed Petri dishes. After the cells were adhered, they were treated with dendrimer conjugates and incubated at $37{ }^{\circ} \mathrm{C}$ under $5 \% \mathrm{CO}_{2}$ for $1 \mathrm{~h}$. After incubation, the cells were washed three times with PBS, then fixed with $4 \%$ formaldehyde at room temperature for 15 min. After fixation, the cells were washed and permeabilized with $0.25 \%$ Triton-X100 for $20 \mathrm{~min}$. The in situ CuAAC reaction was performed on the monolayer of cells by using the same CUAAC protocol as that described above. The cells were then washed twice with $0.1 \%$ BSA/PBS solution and stained with DAPI and ProLong Gold Antifade Reagent (Life Technologies, Carlsbad, CA, USA). Confocal images were obtained on an Olympus FV-500 confocal microscope with a 1003, 1.4 NA oil-immersion objective. To visualize AF488, the $488 \mathrm{~nm}$ line of an argon-ion laser was used for excitation, and the emission was filtered at $505-525 \mathrm{~nm}$. To visualize AF555, the $543 \mathrm{~nm}$ line of a He-Ne laser was used for excitation, and the emission was filtered at $555-625 \mathrm{~nm}$.

\section{RNA isolation, reverse transcription, and real-time poly- merase chain reaction}

After washing the cells, total RNA was isolated and extracted with the RNeasy Mini Kit (Qiagen, Valencia, CA, USA). Total RNA (500 ng) was amplified by using the Message Amp II RNA Kit (Ambion, Austin, TX, USA) and analyzed in triplicate by RT-PCR. Expression was normalized to glyceraldehyde 3-phosphate dehydrogenase and represented as the fold-change over control. Primers were purchased from Life Technologies (Carlsbad, CA, USA).

\section{Acknowledgements}

This project was funded in whole or in part with Federal funds from the National Cancer Institute, National Institutes of Health, under award 1R01 CA119409. S.N.G. was supported by T32L007853 from the National Heart, Lung, and Blood Institute.

Keywords: cancer · click chemistry · drug delivery
inflammation - nanoparticles

[1] R. H. Müller, D. Rühl, M. Lück, B.-R. Paulke, Pharm. Res. 1997, 14, 18-24.

[2] R. Huisgen, Angew. Chem. 1963, 75, 604-637; Angew. Chem. Int. Ed. Engl. 1963, 2. 565-598.

[3] a) H. C. Kolb, M. G. Finn, K. B. Sharpless, Angew. Chem. 2001, 113, 2056 2075; Angew. Chem. Int. Ed. 2001, 40, 2004-2021; b) A. B. Neef, C. Schultz, Angew. Chem. 2009, 121, 1526-1529; Angew. Chem. Int. Ed. 2009, 48, 1498-1500; c) Q. Wang, T. R. Chan, R. Hilgraf, V. V. Fokin, K. B. Sharpless, M. G. Finn, J. Am. Chem. Soc. 2003, 125, 3192-3193; d) Y. Y. Yang, J. M. Ascano, H. C. Hang, J. Am. Chem. Soc. 2010, 132, 3640-3641.

[4] a) J. M. Baskin, J. A. Prescher, S. T. Laughlin, N. J. Agard, P. V. Chang, I. A. Miller, A. Lo, J. A. Codelli, C. R. Bertozzi, Proc. Natl. Acad. Sci. USA 2007, 104, 16793-16797; b) P. V. Chang, X. Chen, C. Smyrniotis, A. Xenakis, T. Hu, C. R. Bertozzi, P. Wu, Angew. Chem. 2009, 121, 4090-4093; Angew. Chem. Int. Ed. 2009, 48, 4030-4033; c) J. A. Prescher, C. R. Bertozzi, Nat. Chem. Biol. 2005, 1, 13-21; d) A. Salic, T. J. Mitchison, Proc. Natl. Acad. Sci. USA 2008, 105, 2415-2420.

[5] a) A. T. Dirks, J. J. Cornelissen, R. J. Nolte, Bioconjugate Chem. 2009, 20, 1129-1138; b) K. Sivakumar, F. Xie, B. M. Cash, S. Long, H. N. Barnhill, Q. Wang, Org. Lett. 2004, 6, 4603-4606.

[6] a) J. F. Kukowska-Latallo, K. A. Candido, Z. Cao, S. S. Nigavekar, I. J. Majoros, T. P. Thomas, L. P. Balogh, M. K. Khan, J. R. Baker, Jr., Cancer Res. 2005, 65, 5317-5324; b) T. P. Thomas, I. J. Majoros, A. Kotlyar, J. F. Kukowska-Latallo, A. Bielinska, A. Myc, J. R. Baker, Jr., J. Med. Chem. 2005, 48, 3729-3735; c) W. Xia, A. R. Hilgenbrink, E. L. Matteson, M. B. Lockwood, J. X. Cheng, P. S. Low, Blood 2009, 113, 438-446; d) A. PuigKroger, E. Sierra-Filardi, A. Dominguez-Soto, R. Samaniego, M. T. Corcuera, F. Gomez-Aguado, M. Ratnam, P. Sanchez-Mateos, A. L. Corbi, Cancer Res. 2009, 69, $9395-9403$.

[7] C. Cullander, Methods Mol. Biol. 1999, 122, 59-73.

[8] T. E. Wileman, M. R. Lennartz, P. D. Stahl, Proc. Natl. Acad. Sci. USA 1986, $83,2501-2505$

[9] a) S. K. Biswas, A. Mantovani, Nat. Immunol. 2010, 11, 889-896; b) A Saccani, T. Schioppa, C. Porta, S. K. Biswas, M. Nebuloni, L. Vago, B. Bottazzi, M. P. Colombo, A. Mantovani, A. Sica, Cancer Res. 2006, 66, $11432-11440$.

[10] a) S. Hong, P. R. Leroueil, I. J. Majoros, B. G. Orr, J. R. Baker, Jr., M. M. Banaszak Holl, Chem. Biol. 2007, 14, 107-115; b) C. Tassa, J. L. Duffner, T. A. Lewis, R. Weissleder, S. L. Schreiber, A. N. Koehler, S. Y. Shaw, Bioconjugate Chem. 2010, 21, 14-19.

[11] J. W. van der Heijden, R. Oerlemans, B. A. Dijkmans, H. Qi, C. J. van der Laken, W. F. Lems, A. L. Jackman, M. C. Kraan, P. P. Tak, M. Ratnam, G. Jansen, Arthritis Rheum. 2009, 60, 12-21.

[12] T. P. Thomas, B. Huang, S. K. Choi, J. E. Silpe, A. Kotlyar, A. M. Desai, H. Zong, J. Gam, M. Joice, J. R. Baker, Jr., Mol. Pharm. 2012, 9, 2669-2676. 


\section{CHEMPLUSCHEM}

[13] a) T. Cedervall, I. Lynch, S. Lindman, T. Berggard, E. Thulin, H. Nilsson, K. A. Dawson, S. Linse, Proc. Natl. Acad. Sci. USA 2007, 104, 2050-2055 b) W. R. Sanhai, J. H. Sakamoto, R. Canady, M. Ferrari, Nat. Nanotechnol. 2008, 3, 242-244.

[14] T. P. Thomas, S. N. Goonewardena, I. J. Majoros, A. Kotlyar, Z. Cao, P. R Leroueil, J. R. Baker, Jr., Arthritis. Rheum. 2011, 63, 2671-2680.

[15] I. J. Majoros, T. P. Thomas, C. B. Mehta, J. R. Baker, Jr., J. Med. Chem 2005, 48, $5892-5899$.

[16] M. T. Islam, I. J. Majoros, J. R. Baker, Jr., J. Chromatogr., B Anal. Technol. Biomed. Life Sci. 2005, 822, 21-26.
[17] D. G. Mullen, M. Fang, A. Desai, J. R. Baker, B. G. Orr, M. M. Banaszak Holl, ACS Nano 2010, 4, 657-670.

[18] H. Zong, T.P. Thomas, K. H. Lee, A. M. Desai, M. H. Li, A. Kotlyar, Y Zhang, P. R. Leroueil, J. J. Gam, M. M. Banaszak Holl, J. R. Baker, Biomacromolecules 2012, 13, 982-991.

[19] S. Manzanero, Methods Mol. Biol. 2012, 844, 177-181.

Received: January 6, 2013

Revised: March 4, 2013

Published online on April 3, 2013 